\title{
Los riesgos de las TIC en las relaciones entre iguales. Cyberbullying en Educación Primaria y Secundaria
}

\section{The risks of ICT in the relationships among peers. Cyberbullying in the Primary and Secondary Education}

\author{
Juan Manuel Rodríguez-Álvarez¹, María Carmen Cabrera Herrera², Santiago Yubero \\ Jiménez ${ }^{3}$ \\ ${ }^{1}$ Consejería de Educación, Cultura y Deporte, Comunidad de Madrid, España (jmrodriguezalvarez@educa.madrid.org) \\ ${ }^{2}$ Área de Intervención Social. Ayuntamiento de Cuenca, España (mccabrera@cuenca.es) \\ ${ }^{3}$ Departamento de Psicología, Universidad de Castilla-La Mancha, España (santiago.yubero@uclm.es)
}

\section{RESUMEN:}

La irrupción de las tecnologías de la información y la comunicación (TIC) en la sociedad han provocado un cambio estructural muy profundo que conlleva nuevas formas de relacionarse. Para poder educar en el uso adecuado y responsable de las tecnologías, desde las enseñanzas regladas establecidas en el sistema educativo, es necesario conocer previamente la implicación de los alumnos en los riesgos que conllevan las tecnologías. El objetivo de este estudio es conocer los comportamientos de victimización y agresión en las conductas de ciberacoso, realizando un análisis comparativo entre estudiantes de Educación Primaria y Educación Secundaria. El estudio se ha realizado en 24 centros con la participación de 2197 alumnos de entre 10 y 18 años. Se ha aplicado una adaptación del Bullying Harassment, and Agression Receipt Measure (Hall, 2016) para su aplicación en España y en los dos roles de participación, víctimas y agresores. Los comportamientos de victimización y agresión son más elevados en Educación Secundaria. Los resultados indican que los comportamientos de cyberbullying se están produciendo ya en los estudiantes de Educación Primaria. Las buenas prácticas con TIC en los contextos escolares deberían tener en cuenta los riesgos potenciales en las relaciones sociales entre iguales. Debería insertarse las medidas preventivas del acoso entre las prioridades pedagógicas.
PALABRAS ClAVE: ACOSO, EDUCACIÓN, RELACIONES ENTRE PARES, TECNOLOGÍA DE LA COMUNICACIÓN

\begin{abstract}
:
The irruption of the information and communication technologies (ICT) in the society has led to a deep structural change that implies new ways to relate. In order to educate in the appropriated and responsible use of the technologies, from the regulated education established in the educative system, it is necessary the previous knowledge of the implication of the pupils and the risks that the technologies imply. The objective of this study is to know the behaviours of victimization and aggression in the cyberbullying behaviours, through a comparative analysis between students of Primary and Secondary School. The study has been developed in 24 educational centres with the participation of 2197 pupils aged between 10 and 18 years old. An adaptation of the Bullying Harassment, and Agression Receipt Measure (Hall, 2016) has been applied in Spain and in the two roles of participation, victims and aggressors. The behaviours of victimization and aggression are higher in Secondary School. The results indicate that the behaviours of cyberbullying are already happening in the students of Primary School. The good practices with ICT in the school contexts should consider the potential risks in the social
\end{abstract}


relationships among equals. The preventive measures should be inserted among the pedagogical priorities.

KEYWORDS: BULLYING, EDUCATION, PEER RELATIONSHIP, TELECOMMUNICATIONS

\section{INTRODUCCIÓN}

La irrupción de las TIC en la sociedad ha provocado un cambio estructural muy profundo en las relaciones sociales y personales. La forma de comunicación es inmediata y la cantidad de contenidos multimedia que se comparten es exponencial en una sociedad electrónica y autodidacta. La edad de inicio en el uso de telefonía móvil es cada vez más temprana y en España, los niños de 2 a 3 años utilizan habitualmente el móvil de sus padres. En 2015 un 98\% de los jóvenes españoles de 10 a 14 años contaba ya con un teléfono de última generación con conexión a Internet (Ditrendia, 2016). El 27\% de niños entre 11 y 14 años de edad reconoce no apagar nunca su móvil. Respecto al uso de las redes sociales, el 66.8\% de los usuarios de Internet han participado en los últimos 3 meses en Facebook, Twitter o Instagram, creando perfiles de usuario y enviando mensajes, siendo los más participativos los estudiantes, con un $90.7 \%$. Para Gabelas, Lazo y Aranda (2012), un entorno TRIC (tecnologías de información relación y comunicación) de prácticas culturales y digitales es una herramienta esencial para la interacción, el aprendizaje y el juego. Aunque conlleva riesgos asociados al tipo y cantidad de relaciones que se generan, el uso indiscriminado de las redes aumenta las posibilidades de que se sufra ciberacoso por la mayor exposición a esas relaciones con sus iguales (Calmaestra et al., 2016; Monks, Ortega, Robinson y Worlidge, 2009). De hecho, el uso del teléfono móvil es la herramienta más habitual para acosar telemáticamente, siendo el WhatsApp la aplicación más utilizada (Fundación ANAR y Mutua Madrileña, 2016).

Se trata de un problema de gran relevancia por las consecuencias que tiene sobre los alumnos que intervienen en los procesos de acoso y por las consecuencias en el ámbito educativo (Lara-Ros, Rodríguez-Jiménez, Martínez-González y Piqueras, 2017; Navarro, Ruiz-Oliva, Larrañaga y Yubero, 2015). Los síntomas psicológicos son mal genio y nerviosismo, sentimientos de soledad e impotencia; físicamente aparecen dolores de cabeza, de estómago, de espalda y vértigo. A nivel escolar, puede producirse bajo rendimiento académico y absentismo escolar (Hernández y Solano, 2007; Ybarra y Mitchell, 2004).

\subsection{Bullying y ciberbullying como concepto}

La definición de bullying más empleada es la de Olweus, que refiere a bullying cuando un alumno está siendo maltratado o victimizado, cuando él o ella está expuesto repetidamente, a lo largo del tiempo, a acciones negativas de otro o de un grupo de estudiantes (Olweus, 1999). El cyberbullying aparece como un nuevo tipo de acoso que se da a través de las TIC para acosar, vejar, insultar, amenazar o intimidar deliberadamente a alguien (Calmaestra et al., 2016). Si bien el bullying se relaciona de forma más directa con el contexto escolar que el cyberbullying, cada vez más estudios muestran correlación entre ambos. Aunque el cyberbullying ocurre a través de los medios digitales, forma parte de la realidad cotidiana de los menores y, por tanto, sería erróneo concebirlos como ámbitos separados e independientes (Fundación ANAR y Mutua Madrileña, 2016). Se trata de una agresión intencional por parte de un individuo o grupo mediante formas electrónicas de contacto, repetidas veces, contra una víctima que no se puede defender con facilidad (Smith et al., 2008). Ambas formas de violencia tienen comportamientos repetitivos de hostigamiento, intimidación y exclusión social. En el bullying, las agresiones suelen ser de tipo físico, psicológico o verbal predominando los insultos y las patadas; en cyberbullying se trata de amenazas, difusión de imágenes comprometidas, difusión de información personal de la víctima y pirateo de las cuentas personales. Aunque el cyberbullying se suele producir en momentos en los que no hay ningún adulto presente en el recinto escolar, o sus inmediaciones, en actividades extraescolares o desde el propio hogar (Calmaestra et al., 2016), la responsabilidad está recayendo sobre el contexto escolar.

\subsection{Inicio del cyberbullying}

El cyberbullying está en constante cambio (Paul, Smith y Blumberg, 2012; Tomczyk, 2017). Los primeros cuestionarios desarrollados sobre el cyberbullying diferenciaban si el comportamiento se producía a través de teléfono o de Internet (Brighi, Guarini y Genta, 2009). Sin embargo, en los últimos años, con la aparición de los móviles con acceso a Internet esta distinción ha perdido el significado.

Aunque la mayoría de los estudios sobre cyberbullying se han desarrollado con alumnos de edades superiores a 11 años, los niños menores de 
11 años también emplean internet y teléfonos móviles (Byron Review, 2008; Carphone Warehouse, 2006). Monks et al. (2009) encuentran que un $72 \%$ de los menores entre 7 y 11 años tienen móvil y el $87 \%$ accede a internet en sus casas. El Anti-Bullying Alliance (ABA, 2009) encontró que un $20 \%$ de escolares entre 10 y 11 años eran víctimas de cyberbullying. Resultados similares fueron informados por Monks et al. (2009) en escolares entre 7 y 11 años: $5 \%$ de agresores y $23 \%$ de víctimas. Sakellariou, Carroll y Houghton (2012) también informaron de comportamientos de cyberbullying entre los 9 y los 11 años en Australia. Cada vez son más los estudios que coinciden en que los comportamientos de acoso y ciberacoso se producen en las dos etapas educativas (Garaigordobil, 2015; Monks, Robinson y Worlidge, 2012; Pérez y Vicario-Molina, 2016).

A nivel internacional, un estudio con 398 estudiantes israelíes (Olenik-Shemesh y Heiman, 2014) indican una victimización de cyberbullying del 20,4\% en la etapa de Educación Primaria.

Existen pocos estudios nacionales sobre la incidencia del cyberbullying en Primaria, existiendo discrepancia entre ellos (del Río, del Barco, Castaño y Carroza, 2014). Por ejemplo, en Murcia, con estudiantes de $5^{\circ}$ y $6^{\circ}$ de Educación Primaria, Giménez, Arnaiz y Maquilón (2013) informan de un porcentaje de ciberagresión del 12,3\% y de cibervictimización del 17,8\%. González y Calvo (2012) analizan el riesgo percibido de ser víctima o agresor de cyberbullying en los estudiantes de la misma región. Los alumnos de $5^{\circ}$ de Educación Primaria perciben un riesgo superior, $32 \%$, a los alumnos de $6^{\circ}$, con un $13,1 \%$. Es necesario incrementar el estudio de la población escolar que se encuentra al final de la etapa de Educación Primaria (Giménez, 2015).

El presente estudio se dirige a conocer la incidencia de los comportamientos de acoso, especialmente de cyberbullying, en los alumnos de los dos últimos cursos de Educación Primaria y en la Educación Secundaria Obligatoria, tanto en los roles de víctima como de agresor. En segundo lugar, queremos analizar las diferencias en los comportamientos de acoso entre los dos niveles educativos.

\section{MATERIAL Y MÉTODO}

\subsection{Participantes}

Han participado 2197 alumnos, el 51,4\% (1130) de Educación Primaria y el 48,6\% (1067) de Educación Secundaria. La edad de los participantes se encuentra entre 10 y 18 años, con una media de 12,93 años.

\subsection{Instrumentos}

- Cuestionario de Acoso y Cyberbullying. Se ha aplicado una adaptación del Bullying Harassment, and Agression Receipt Measure (Hall, 2016) para su aplicación en España y en los dos roles de participación: víctimas y agresores. Consta de 14 ítems que describen los comportamientos de acoso (físico, verbal y exclusión, del 1 al 11) y de cyberbullying (del 12 al 14). En las tablas pueden observarse los ítems de las escalas. El alumno debía informar sobre la frecuencia en que había recibido y/o realizado durante el último mes los comportamientos de acoso de que consta la escala. La escala de respuesta es una tipo Likert de cuatro anclajes: 0- No me ha ocurrido, 1- Me ha ocurrido 1 o 2 veces, 2- Me ha ocurrido al menos 1 vez a la semana, 3- Me ha ocurrido 2 o más veces a la semana. La fiabilidad en la escala de victimización, según el alfa de Cronbach, es $\alpha=0,87$ y en la escala de agresión es $\alpha=0,81$.

\subsection{Procedimiento}

Se estableció contacto por vía telefónica y mediante correo electrónico con los equipos directivos y/o los departamentos de orientación de diversos centros tanto de Castilla-La Mancha como de la Comunidad de Madrid, de entornos rurales y urbanos, seleccionados al azar. De ellos, se obtuvo respuesta afirmativa de 24 centros.

Previa autorización de los padres, los cuestionarios fueron pasados en las aulas de los centros durante el horario lectivo.

\subsection{Análisis de datos}

En primer lugar, para conocer la incidencia de los comportamientos de acoso, se han calculado las frecuencias de participación en los comportamientos de bullying y cyberbullying evaluados, tanto para la victimización como para la agresión. Posteriormente, se han analizado las diferencias de la implicación en cada uno de los comportamientos de bullying y cyberbullying entre los alumnos de Primaria y de Secundaria, a través de un contrate de medias empleando la $t$ de Student. 


\section{RESULTADOS}

\subsection{Incidencia de los comportamientos de acoso}

Como se puede apreciar en la tabla 1, la incidencia de los comportamientos de victimización de cyberbullying (ítems 12, 13 y 14) son menores que en los comportamientos de bullying. En torno al 5\% del alumnado de Primaria y Secundaria ha sido cibervíctimizado.

El comportamiento más frecuente es haber recibido insultos o motes, un $64 \%$ informa que no le ha ocurrido, $8 \%$ informa que le ha pasado más de dos veces a la semana. Casi un 5\% informa de victimización intensa, más de dos veces a la semana, en que le dijeron algo dañino y que trataron de poner a la gente en su contra. Los porcentajes más elevados de participación se encuentran en la ocurrencia puntual, una o dos veces. En este caso, se incluyen los comportamientos de difusión de rumores y las acciones físicas: le han empujado, le han lanzado algo y han chocado contra él/ella.

Como ya hemos comentado, los comportamientos de cyberbullying son informados por menos alumnos, un $97 \%$ informó que nunca habían colgado nada malo sobre él/ella en Internet, un $94 \%$ que no le habían enviado ningún mensaje dañino y un $95 \%$ indicó que nunca habían hecho comentarios dañinos en Internet.

Tabla 1: Comportamientos de victimización, en porcentajes

\begin{tabular}{|c|c|c|c|c|}
\hline Ítems & 0 & 1 & 2 & 3 \\
\hline $\begin{array}{l}\text { 1-Me han empujado o tirado } \\
\text { de mí. }\end{array}$ & 71.8 & 22.2 & 3.4 & 2.6 \\
\hline 2-Me han lanzado algo. & 68.8 & 23.8 & 4.8 & 2.6 \\
\hline $\begin{array}{l}\text { 3-Han chocado contra mí o } \\
\text { me han hecho tropezar. }\end{array}$ & 66.4 & 24.4 & 6.2 & 3.0 \\
\hline $\begin{array}{l}\text { 4-Me han dado un puñetazo o } \\
\text { me han abofeteado. }\end{array}$ & 89.4 & 7.9 & 1.6 & 1.1 \\
\hline $\begin{array}{l}\text { 5-Me han atacado con algún } \\
\text { objeto. }\end{array}$ & 91.6 & 6.4 & 1.2 & 0.9 \\
\hline $\begin{array}{l}\text { 6-Me insultaron o pusieron } \\
\text { motes. }\end{array}$ & 64.1 & 23.0 & 5.1 & 7.9 \\
\hline 7-Me dijeron algo dañino. & 67.3 & 21.7 & 6.1 & 4.9 \\
\hline $\begin{array}{l}\text { 8-Me dijeron algo para } \\
\text { asustarme o intimidarme. }\end{array}$ & 81.0 & 13.9 & 3.1 & 2.0 \\
\hline $\begin{array}{l}\text { 9-Trataron de poner a la } \\
\text { gente en mi contra. }\end{array}$ & 71.5 & 19.3 & 4.6 & 4.6 \\
\hline $\begin{array}{l}\text { 10-Difundieron falsos } \\
\text { rumores sobre mí. }\end{array}$ & 69.7 & 21.0 & 5.3 & 3.9 \\
\hline 11-Me excluyeron del grupo. & 81.4 & 13.0 & 2.7 & 2.9 \\
\hline $\begin{array}{l}\text { 12-Han colgado algo malo } \\
\text { sobre mí en Internet. }\end{array}$ & 97.1 & 1.9 & 0.4 & 0.6 \\
\hline 13-Me han enviado un email & 94.2 & 4.5 & 0.6 & 0.7 \\
\hline
\end{tabular}

o un mensaje dañino.

14-Han hecho comentarios $\quad 95.1 \quad 3.5 \quad 0.7 \quad 0.6$ dañinos acerca de mí en

Internet.

Nota: $0=$ No me ha ocurrido; $1=$ Me ha ocurrido 1 o 2 veces; $2=$ Me ha ocurrido al menos $1 \mathrm{vez}$ a la semana; $3=$ Me ha ocurrido 2 o más veces a la semana.

Respecto de los comportamientos de agresión (tabla 2), también son los comportamientos de ciberagresión los menos informados (ítems 12, 13 y 14). Entre el 2 y el 3\% del alumnado de Primaria y de Secundaria informó ser ciberagresor. El comportamiento de acoso más frecuente es insultar o poner motes $(30 \%)$.

Como puede observarse, los porcentajes que informan de agresión son mucho más bajos que los comportamientos informados de victimización. Casi un $99 \%$ informó de no haber colgado nunca nada malo sobre alguien en Internet. Más del 97\% indicó no haber realizado ni enviado comentarios dañinos en Internet. Aunque la incidencia sea baja, es importante destacar la realización de comportamientos de agresión empleando las nuevas tecnologías, el envío de emails y mensajes dañinos fue informado por un $0,4 \%$ que lo habían realizado dos o más veces a la semana.

El comportamiento de acoso que informan realizar con más frecuencia es insultar o poner motes, un $30 \%$ lo ha realizado en alguna ocasión, un $2 \%$ más de dos veces a la semana ha insultado a un compañero.

En torno a un 1\% también ha lanzado algo a un compañero, ha chocado contra alguien y ha dicho algo dañino, más de dos veces a la semana.

El comportamiento menos informado de agresión de acoso es la violencia física de ataque con un objeto, pero hay que tener en cuenta que casi un $4 \%$ indicó que lo había realizado una o dos veces, y un $0,7 \%$ con mayor frecuencia.

Tabla 2: Comportamientos de agresión, en porcentajes

\begin{tabular}{lllll}
\hline Ítems & 0 & 1 & 2 & 3 \\
\hline $\begin{array}{l}\text { 1-He empujado o he tirado de } \\
\text { alguien. }\end{array}$ & 82.0 & 15.1 & 2.0 & 0.9 \\
$\begin{array}{l}\text { 2-He lanzado algo a otro } \\
\text { estudiante. }\end{array}$ & 85.1 & 11.9 & 1.9 & 1.1 \\
$\begin{array}{l}\text { 3-He chocado contra alguien } \\
\text { o le he hecho tropezar. }\end{array}$ & 80.7 & 15.9 & 2.1 & 1.3 \\
$\begin{array}{l}\text { 4-He dado un puñetazo o he } \\
\text { abofeteado a alguien. }\end{array}$ & 91.4 & 6.9 & 1.0 & 0.6 \\
$\begin{array}{l}\text { 5-He atacado a alguien con } \\
\text { un objeto. }\end{array}$ & 95.5 & 3.9 & 0.5 & 0.2 \\
$\begin{array}{l}\text { 6-He insultado o puesto } \\
\text { motes a alguien. }\end{array}$ & 70.9 & 24.2 & 2.9 & 2.0 \\
$\begin{array}{l}\text { 7-He dicho algo dañino a } \\
\text { alguien. }\end{array}$ & 80.3 & 16.7 & 1.8 & 1.2 \\
\hline
\end{tabular}




\begin{tabular}{lcccc}
\hline $\begin{array}{l}\text { 8-He dicho algo para asustar } \\
\text { o intimidar a alguien. }\end{array}$ & 91.4 & 7.1 & 1.1 & 0.5 \\
$\begin{array}{l}\text { 9-He tratado de poner a otra } \\
\text { persona en contra de alguien. }\end{array}$ & 91.6 & 7.0 & 1.1 & 0.4 \\
$\begin{array}{l}\text { 10-He difundido falsos } \\
\text { rumores sobre alguien. }\end{array}$ & 95.1 & 4.3 & 0.4 & 0.2 \\
$\begin{array}{l}\text { 11-He excluido a alguien del } \\
\text { grupo. }\end{array}$ & 92.5 & 6.3 & 0.7 & 0.5 \\
$\begin{array}{l}\text { 12-He colgado e Internet algo } \\
\text { malo sobre alguien. }\end{array}$ & 98.8 & 1.0 & 0.1 & 0.1 \\
$\begin{array}{l}\text { 13-He enviado a alguien un } \\
\text { email o mensaje dañino. }\end{array}$ & 97.6 & 1.8 & 0.2 & 0.4 \\
$\begin{array}{l}\text { 14-He hecho comentarios } \\
\text { dañinos acerca de alguien en }\end{array}$ & 97.7 & 1.8 & 0.2 & 0.2 \\
Internet. & & & \\
\hline $\begin{array}{l}\text { Nota: } 0=\text { No me ha ocurrido; } 1=\text { Me ha ocurrido 1 o 2 veces; } 2= \\
\text { Me ha ocurrido al menos 1 vez a la semana; } 3=\text { Me ha ocurrido } \\
\text { 2 o más veces a la semana. }\end{array}$
\end{tabular}

\subsection{Análisis comparativo entre Educación Primaria y Educación Secundaria}

Como puede apreciarse en las tablas 3 y 4, los comportamientos de bullying y cyberbullying se incrementan ligeramente de Primaria a Secundaria, pero todos los comportamientos tienen su aparición en el periodo analizado de Educación Primaria. En la comparación de la victimización (tabla 3), se producen diferencias significativas en los últimos 3 ítems de cyberbullying. Sin embargo, al analizar el comportamiento de ciberagresión (tabla 4), solamente se mantiene para el último ítem.

Tabla 3: Comparación de medias en la victimización entre Educación Primaria y Educación Secundaria

\begin{tabular}{lcccc}
\hline Ítems & E. Primaria & E. Secundaria & & \\
& $M(D T)$ & $M(D T)$ & $t$ & $p$ \\
\hline 1-Me han empujado o tirado de mí. & $0.36(0.68)$ & $0.38(0.67)$ & -0.59 & .554 \\
2-Me han lanzado algo. & $0.32(0.61)$ & $0.51(0.77)$ & -6.38 & .000 \\
3-Han chocado contra mí o me han hecho tropezar. & $0.46(0.77)$ & $0.45(0.72)$ & 0.33 & .738 \\
4-Me han dado un puñetazo o me han abofeteado. & $0.16(0.49)$ & $0.12(0.45)$ & 1.81 & .069 \\
5-Me han atacado con algún objeto. & $0.12(0.42)$ & $0.11(0.42)$ & 0.32 & .748 \\
6-Me insultaron o pusieron motes. & $0.59(0.95)$ & $0.54(0.85)$ & 1.14 & .257 \\
7-Me dijeron algo dañino. & $0.50(0.85)$ & $0.47(0.77)$ & 1.14 & .255 \\
8-Me dijeron algo para asustarme o intimidarme. & $0.25(0.60)$ & $0.27(0.63)$ & -0.94 & .350 \\
9-Trataron de poner a la gente en mi contra. & $0.43(0.82)$ & $0.41(0.74)$ & 0.49 & .624 \\
10-Difundieron falsos rumores sobre mí. & $0.37(0.73)$ & $0.50(0.80)$ & -4.03 & .000 \\
11-Me excluyeron del grupo. & $0.30(0.69)$ & $0.24(0.61)$ & 1.85 & .064 \\
12-Han colgado algo malo sobre mí en Internet. & $0.02(0.23)$ & $0.06(0.36)$ & -3.45 & .001 \\
13-Me han enviado un email o un mensaje dañino. & $0.05(0.29)$ & $0.10(0.41)$ & -3.19 & .001 \\
14-Han hecho comentarios dañinos acerca de mí en Internet. & $0.05(0.29)$ & $0.09(0.39)$ & -3.04 & .002 \\
\hline
\end{tabular}

Nota: Escala de medida de 0 a 3

Tabla 4: Comparación de medias en la agresión entre Educación Primaria y Educación Secundaria

\begin{tabular}{lcccc}
\hline Ítems & E. Primaria & E. Secundaria & & \\
& $M(D T)$ & $M(D T)$ & $t$ & $p$ \\
\hline 1-He empujado o he tirado de alguien. & $0.19(0.47)$ & $0.24(0.55)$ & -2.39 & .017 \\
2-He lanzado algo a otro estudiante. & $0.11(0.38)$ & $0.28(0.61)$ & -7.91 & .000 \\
3-He chocado contra alguien o le he hecho tropezar. & $0.21(0.51)$ & $0.27(0.60)$ & -2.72 & .007 \\
4-He dado un puñetazo o he abofeteado a alguien. & $0.11(0.39)$ & $0.11(0.40)$ & -0.33 & .741 \\
5-He atacado a alguien con un objeto. & $0.05(0.27)$ & $0.64(0.28)$ & -1.46 & .144 \\
6-He insultado o puesto motes a alguien. & $0.32(0.61)$ & $0.40(0.66)$ & -3.29 & .001 \\
7-He dicho algo dañino a alguien. & $0.22(0.52)$ & $0.26(0.56)$ & -1.88 & .060 \\
8-He dicho algo para asustar o intimidar a alguien. & $0.10(0.38)$ & $0.12(0.38)$ & -1.08 & .281 \\
9-He tratado de poner a otra persona en contra de alguien. & $0.11(0.40)$ & $0.09(0.34)$ & 1.07 & .285 \\
10-He difundido falsos rumores sobre alguien. & $0.06(0.27)$ & $0.57(0.28)$ & 0.25 & .806 \\
11-He excluido a alguien del grupo. & $0.11(0.40)$ & $0.07(0.30)$ & 2.80 & .005 \\
12-He colgado e Internet algo malo sobre alguien. & $0.01(0.13)$ & $0.02(0.18)$ & -1.82 & .069 \\
13-He enviado a alguien un email o mensaje dañino. & $0.03(0.22)$ & $0.04(0.27)$ & -1.08 & .281 \\
14-He hecho comentarios dañinos acerca de alguien en Internet. & $0.02(0.16)$ & $0.04(0.25)$ & -2.26 & .024 \\
\hline
\end{tabular}

Nota: Escala de medida de 0 a 3

Las víctimas de Secundaria informan de mayor difusión de rumores que en la etapa de Primaria, aunque en la agresión de exclusión en Primaria aparece más elevado el comportamiento de excluir a alguien del grupo. En Secundaria se incrementa la agresión a través de insultos y motes, también 
las formas directas de acoso de empujar y chocar/hacer tropezar contra un compañero. El lanzar algo se incrementa tanto en la agresión como en la victimización.

En cinco comportamientos de acoso no se han encontrado diferencias significativas en agresión ni en victimización entre los alumnos de Primaria y los alumnos de Secundaria, en la agresión física directa de dar un puñetazo y atacar con un objeto, en las agresiones verbales de decir algo dañino e intimidar y poner a otra persona en contra,

\section{DISCUSIÓN}

Nuestro objetivo era conocer la incidencia de las conductas de cyberbullying, desde un análisis comparativo entre los estudiantes de Educación Primaria y Secundaria.

Los resultados coinciden con las investigaciones previas (Garaigordobil, 2015; Pérez y Vicario-Molina, 2016) indicando que el cyberbullying es un problema desde las primeras etapas educativas. De acuerdo con los estudios nacionales en estudiantes de Educación Primaria (Gimenez, 2015; Giménez, Arnaiz y Maquilón, 2013; González y Calvo, 2012), los comportamientos de cyberbullying, tanto de agresión como de acoso, aparecen en los dos últimos cursos de Primaria.

Nuestros resultados son similares a los de Sakellariou et al. (2012), que encontraron niveles más elevados de cibervictimización entre los 11 y 14 años, que en los alumnos de Primaria, entre 9 y 11 años. En la ciberagresión los alumnos mayores mostraron mayor implicación que los alumnos de primaria. Resultados coincidentes con los informados por Garaigordobil (2015) que encontró un aumento de agresores a medida que aumenta la edad en estudiantes de 12 a 18 años.

Nos parece de especial relevancia indicar, que ya desde la etapa de Educación Primaria se produzcan comportamientos de cyberbullying, especialmente que hagan uso de Internet para hacer comentarios dañinos sobre los compañeros. Podríamos interpretar que el cyberbullying es una extensión del bullying en el que se emplean las TIC en las interacciones sociales. Las buenas prácticas con TIC en los contextos escolares deberían tener en cuenta los riesgos potenciales en las relaciones sociales entre iguales.

Teniendo en cuenta que uno de los principales usos que realizan los estudiantes de Educación Primaria y Secundaria de las TIC es relacional, las intervenciones educativas tienen que incluir también pautas de utilización adecuadas. Las TIC pueden provocar un proceso de victimización y agresión de consecuencias muy perjudiciales para los escolares.

Debemos hacerles conscientes que todos los mensajes producidos a través de Internet, tienen características de permanencia y extensión que pueden generar consecuencias negativas para todos los implicados.

Para prevenirlo, se considera necesario el establecimiento de programas de actuación desde edades tempranas y desde un uso respetuoso de las TIC en todos sus ámbitos. Entre todos debemos lograr que la sociedad tenga tolerancia cero contra cualquier tipo de ciberacoso, detectando lo antes posible tanto a las víctimas como a los agresores y minimizando el riesgo que entraña esta nueva forma de agresión digital enmascarada bajo el anonimato de la tecnología.

Debería insertarse las medidas preventivas del cyberbullying entre las prioridades pedagógicas (Tomczyk, 2017). Con el objetivo de conseguir una sociedad electrónica que sea capaz de usar la tecnología para fines positivos, diversos autores sugieren establecer las líneas de actuación desde la prevención e intervención en las primeras etapas del sistema educativo (Garaigordobil, 2015). Estamos de acuerdo con Casas, del Rey y OrtegaRuiz (2016), que la intervención debe realizarse desde el uso relacional de Internet que realizan los niños/as y adolescentes.

Además, como Internet es una extensión del mundo social real de los jóvenes, las medidas para reducir las conductas de riesgo de Internet, como el cyberbullying, requieren también la implicación de los padres (Cross et al., 2016; Diamanduros y Downs, 2011; Larrañaga, Yubero, Navarro y Ovejero, 2016; Morgan, 2013). Muchos padres precisan de apoyo institucional para conocer las posibilidades de empleo y riesgos de internet y para proteger a sus hijos del cyberbullying (Floros, Siomos, Fisoun, Dafouli y Geroukalis, 2013).

Es imprescindible acompañar el desarrollo tecnológico de desarrollo ético (Garaigordobil, 2011) para garantizar la evolución adecuada de los jóvenes en nuestra sociedad y evitar los riesgos que pueden presentar las TIC en las relaciones entre iguales. Consideramos preciso que todas las Comunidades Autónomas puedan contar con respaldo normativo a partir de un protocolo unificado de respuestas educativas ante las situaciones de cyberbullying (Cerezo y Rubio, 2017; Fundación ANAR y Mutua Madrileña, 2016). 
Entre las limitaciones del estudio debemos considerar que algunos estudios han mostrado diferencias entre la titularidad del centro, pública o privada (Machimbarrena y Garaigordobil, 2017). En este estudio no se ha analizado esta variable contextual. Sería interesante estudiar la evolución de Primaria a Secundaria atendiendo al contexto escolar de titularidad del centro. Por otra parte, se trata de un estudio transversal, con sujetos diferentes en cada nivel analizado. Sería necesario replicar el estudio con metodología longitudinal para analizar los procesos evolutivos en la implicación en los comportamientos de acoso.

\section{CONCLUSIONES}

Nuestros resultados confirman que el cyberbullying aparece en Educación Primaria, no siendo exclusivo de Secundaria. Informan de mayor cibervictimización (en torno al 5\%) que ciberagresión (cerca del 3\%). Los alumnos de Secundaria informan de más victimización que los alumnos de Primaria, aunque la agresión informada solamente es superior en realizar comentarios dañinos en Internet.

Respecto a las formas tradicionales de acoso, la agresión física directa con un puñetazo o con un objeto es la menos informada tanto en victimización como en agresión, en los dos ciclos de enseñanza. Por el contrario, la estrategia de acoso que se produce con más frecuencia es el insulto y los motes, también aparecen desde los últimos años de Primaria, aunque se incrementa significativamente en Secundaria.

Los alumnos de Educación Primaria presentan mayor agresión directa de exclusión, excluir a alguien del grupo. En Educación Secundaria los alumnos informan de recibir más acoso de exclusión indirecto, difusión de rumores.

En resumen, el cyberbullying es un problema desde las primeras etapas educativas, apareciendo ya en los dos últimos cursos de Primaria. Por otra parte, también es importante destacar que se produce un aumento de agresiones de acoso en la etapa de Educación Secundaria.

\section{Agradecimientos}

Esta investigación forma parte del Proyecto I+D+i PSI2015-70822-R, "La protección de las víctimas del cyberbullying: un estudio de las variables familiares y de género".

\section{REFERENCIAS}

ABA (2009). New research on cyberbullying highlights the role of parents in revention. Recuperado de http://www.anti-

bullyingalliance.org.uk/press_centre/news_archive/n ew_research_on_cyberbullying.aspx

Brighi, A., Guarini, A. y Genta, M. L. (2009). Bullying in the digital age. En M. L. Genta, A. Brighi y A. Guarini (Eds), Bullying and cyberbullying in adolescence (pp. 14-39). Rome: Carocci.

Byron Review (2008). Safer children in a digital world: The report of the Byron Review. Recuperado de http://www.dcsf.gov.uk/byronreview/pdfs/Final\%20 Report\%20Bookmarked.pdf

Calmaestra, J., Escorial, A., García, P., Del Moral, C., Perazzo, C. y Ubrich, T. (2016). Yo a eso no juego. Madrid: Save the Children Recuperado de https://www.savethechildren.es/sites/default/files/im ce/docs/yo_a_eso_no_juego.pdf

Casas, J. A., del Rey, R. y Ortega-Ruiz, R. (2016). Entornos virtuales: beneficios y riesgos entre los adolescentes y jóvenes. En S. Yubero, E. Larrañaga y R. Navarro (eds.), La violencia en las relaciones humanas: contextos y entornos protectores del menor (pp. 7592). Cuenca: Ediciones de la Universidad de Castilla-La Mancha.

Cerezo, F. y Rubio, F. J. (2017). Medidas relativas al acoso escolar y ciberacoso en la normativa autonómica española. Un estudio comparativo. Revista Electrónica Interuniversitaria de Formación del Profesorado, 20, 113-126.

Cross, D., Shaw, T., Hadwen, K., Cardoso, P., Slee, P., Roberts, C., ... Shaw, T. (2016). Longitudinal impact of the cyber friendly schools program on adolescents' cyberbullying behavior. Aggressive Behavior, 42(2), 166-180.

Del Río, M. I. P., del Barco, B. L., Castaño, E. F. y Carroza, T. G. (2014). Cyberbullying en tercer ciclo de Educación Primaria: variables moduladoras y consecuencias sobre la ansiedad. Apuntes de Psicología, 32(1), 5-14.

Diamanduros, T. y Downs, E. (2011). Creating a safe school environment: Howto prevent cyberbullying at your school. Library Media Connection, 30(2), 36-38.

Ditrendia (2016). Informe Ditrendia: mobile en España y en el mundo. Recuperado de http://www.amic.media/media/files/file_352_1050.p df

Floros, G. D., Siomos, K. E., Fisoun, V., Dafouli, E. y Geroukalis, D. (2013). Adolescent online cyberbullying in Greece: The impact of parental online security practices, bonding, and online impulsiveness. Journal of School Health, 83(6), 445-453.

Fundación ANAR, Fundación Mutua Madrileña. (2016). Acoso escolar: I Estudio sobre el "bullying” según los afectados y líneas de actuación. Recuperado de http://www.observatoriodelainfancia.es/oia/esp/desc argar.aspx $?$ id $=4998 \&$ tipo $=$ documento

Gabelas, J. A., Marta-Lazo, C. y Aranda, D. (2012). Por qué las TRIC y no las TIC. Revista de los Estudios de Ciencias de la Información y de la Comunicación. Recuperado http://www.uoc.edu/divulgacio/comein/es/numero09 /articles/Article-Dani-Aranda.html 
Garaigordobil, M. (2011). Prevalencia y consecuencias del cyberbullying: una revisión. International Journal of Psychology and Psychological Therapy, 11, 233254.

Garaigordobil, M. (2015). Cyberbullying en adolescentes y jóvenes del País Vasco: cambios con la edad. Anales de Psicología, 31(3), 1069-1077.

Giménez, A. M. (2015). Estrategias de afrontamiento ante el cyberbullying. Una mirada cualitativa desde la perspectiva de los escolares. Campo Abierto, monográfico, 49-65.

Giménez, A. M., Arnaiz, P. y Maquilón, J. J. (2013). Cyberbullying, la nueva cara de la violencia escolar. Experiencias en una muestra de estudiantes de Educación Primaria de la Región de Murcia. Madrid: Editorial Académica Española.

González, V. y Calvo, A. R. (2012). Ciberbullying en las aulas de primaria de Murcia. Recuperado de http://www.uoc.edu/divulgacio/comein/es/numero09 /articles/Article-Dani-Aranda.html

Hall, W. J. (2016). Initial development and validation of the Bullyharm: The Bullying, Harassment, and Aggression Receipt Measure. Psychology in the Schools, 53(9), 984-1000.

Hernández, M. A., Solano, I. M. (2007). Ciberbullying, un problema de acoso escolar. Revista Iberoamericana de Educación a Distancia, 10, 17-36.

Lara-Ros, M., Rodríguez-Jiménez, T., Martínez-González, A. E. y Piqueras, J. A. (2017). Relación entre el bullying y el estado emocional en niños de educación primaria. Revista de Psicología Clínica con Niños y Adolescentes, 4(1), 59-64.

Larrañaga, E., Yubero, S., Navarro, R y Ovejero, A. (2016). Loneliness, parent-child communication and cyberbullying victimization among Spanish youths. Computers in Human Behavior, 65, 1-8.

Machimbarrena, J. M. y Garaigordobil, M. (2017). Bullying/Cyberbullying en quinto y sexto curso de primaria: diferencias entre centros públicos y privados. Anales de Psicología, 33(2), 319-326.

Monks, C. P., Ortega, R., Robinson, S. y Orlidge, P. (2009). Cyberbullying among primary school-aged pupils. Kwartalnik Pedagogiczny, 4, 167-181.

Monks, C. P., Robinson, S. y Worlidge, P. (2012). The emergence of cyberbullying: A survey of primary school pupils' perceptions and experiences. School Psychology International, 33(5), 477-491.

Morgan, H. (2013). Malicious use of technology: What schools, parents, and teachers can do to prevent cyberbullying. Childhood Education, 89(3), 146151.

Navarro, R., Ruiz-Oliva, R., Larrañaga, E. y Yubero, S. (2015). The impact of cyberbullying and social bullying on optimism, global and school-related happiness and life satisfaction among 10-12-yearold schoolchildren. Applied Research in Quality of Life, 10(1), 15-36.

Olenik-Shemesh, D., y Heiman, T. (2014). Exploring Cyberbullying among Primary children in relation to social support, loneliness, self-Efficacy, and wellbeing. Child Welfare, 93(5), 27-35.

Olweus, D. (1999). Sweden. En P. K. Smith, Y. Morita, J. Junger-Tas, D. Olweus, R. Catalano y P. Slee (Eds.), The Nature of School Bullying: A cross-national Perspective (pp. 7-27). London: Routledge.

Paul, S., Smith, P. K. y Blumberg, H. H. (2012). Revisiting cyberbullying in schools using the quality circle approach. School Psychology International, 33(5), 492-504.

Pérez, B. y Vicario-Molina, I. (2016). Ciberbullying en la Educación Primaria: análisis de la incidencia de la victimización y la búsqueda de apoyo. En J. L.Castejón (coord.), Psicología y educación: presente y futuro. (pp 2421-2429). Madrid: Asociación Científica de Psicología y Educación.

Sakellariou, T., Carroll, A., y Houghton, S. (2012). Rates of cyber victimization and bullying among male Australian primary and high school students. School Psychology International, 33(5), 533-549.

Smith, P. K. (2016). Bullying escolar. En S. Yubero (Ed.), E. Larrañaga (Ed.) y R. Navarro (Ed.), La violencia en las relaciones humanas: contextos y entornos protectores del menor (pp. 9-29). Cuenca: Ediciones de la Universidad de Castilla-La Mancha.

Smith, P. K., Mahdavi, J., Carvalho, M., Fisher, S., Russell, S. y Tippett, N. (2008). Cyberbullying: Its nature and impact in secondary school pupils. Journal of Child Psychology and Psychiatry and Allied Disciplines, 49, 376-385.

Tomczyk, L. (2017). Cyberbullying in 2010 and 2015- A perspective on the changes in the phenomenon among adolescents in Poland in the context of preventive action. Children and Youth Services Review, 75, 50-60.

Warehouse, C. (2006). Mobile life youth report: The impact of the mobile phone on the lives of young people. Recuperado http://www.mobilelife2008.co.uk/mobilelife2007

Ybarra, M. L. y Mitchell K. J. (2004). Online aggressor/targets, aggressors, and targets: A comparison of associated youth characteristics. Journal of Child Psychology and Psychiatry, 45, 1308-1316. 